\title{
Detection of SARS-CoV-2 variants in India from UK returnees
}

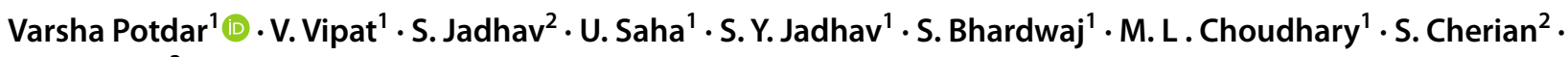 \\ P. Abraham ${ }^{3}$
}

Received: 18 January 2021 / Accepted: 23 April 2021 / Published online: 23 June 2021

(c) Springer-Verlag GmbH Germany, part of Springer Nature 2021, corrected publication 2021

Sir,

The epidemiology of the severe acute respiratory syndrome coronavirus 2 (SARS-CoV-2) has been marked by the emergence of new variants globally. The diversification is normal as a part of the evolutionary and adaptation processes. Most emerging mutations and variants are not known to have any major impact on the spread of the virus. Some mutations may however give a selective advantage to the virus-like change in virulence, transmissibility etc. Among the variants noted in the recent past, a new SARS-CoV-2 variant, VOC 202012/01, was reported from United Kingdom (UK) that was noted to have emerged in late September 2020 [1]. This variant resulted in an increasing proportion of cases in Kent, a county in south-eastern England, London and other parts of the UK and was reported in early December as a variant of concern. Preliminary analyses have indicated that the new variant has increased transmissibility compared to previously circulating variants, with no increase in infection severity. Since late December 2020, a few VOC 202012/01 cases have also been reported in other European countries such as Belgium, Denmark, Finland, France, Germany, Switzerland, Iceland, Ireland, Italy, the Netherlands, Norway, Portugal, Spain and Sweden and globally in countries

\footnotetext{
Varsha Potdar

potdarvarsha9@gmail.com

V. Vipat

veenavipat@gmail.com

S. Jadhav

smjniv@gmail.com

U. Saha

diyasaha95@gmail.com

S. Y. Jadhav

sheetalk86@gmail.com

S. Bhardwaj

sumitduttbhardwaj@gmail.com

M. L . Choudhary

choudhary.ml@gov.in
}

including Australia, Canada, Hong Kong SAR, Israel, Japan, Jordan, Lebanon, South Korea, and Singapore.

Genomic analysis revealed that this new variant of SARS CoV-2 in a lineage named as B.1.1.7 branched-out from the rest of the phylogenetic tree [2]. The U.K. lineage of SARSCoV-2 has picked 17 mutations that lead to 14 amino acid changes in multiple proteins and 3 deletions [2]. Crucial mutations were in the gene that encodes the spike, a protein on the viral surface that the pathogen uses to enter human cells.

The Government of India (GoI) had suspended air travel from the UK since December 23, 2020, in order to check the influx of the new variant of SARS-CoV-2 into the country. Further, to monitor the spread of the UK variant in the country, GoI released a guideline wherein the respective State governments need to ensure that all passengers travelling from or transiting through airports in the UK and disembarking in India need be subjected to the RT-PCR test for SARS-CoV-2 on arrival. Further on, the samples of passengers testing positive should be sent to the Indian Council of Medical Research-National Institute of Virology (ICMRNIV), Pune or any other appropriate laboratory for genomic sequencing. December 23, 2020 onwards, ICMR-NIV, Pune started receiving samples of the COVID-19 positive UK

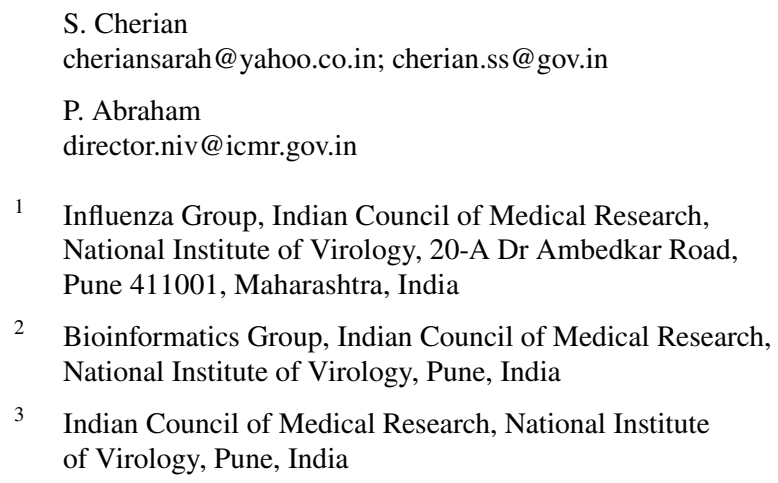

1 Influenza Group, Indian Council of Medical Research, National Institute of Virology, 20-A Dr Ambedkar Road, Pune 411001, Maharashtra, India

2 Bioinformatics Group, Indian Council of Medical Research, National Institute of Virology, Pune, India

3 Indian Council of Medical Research, National Institute of Virology, Pune, India 


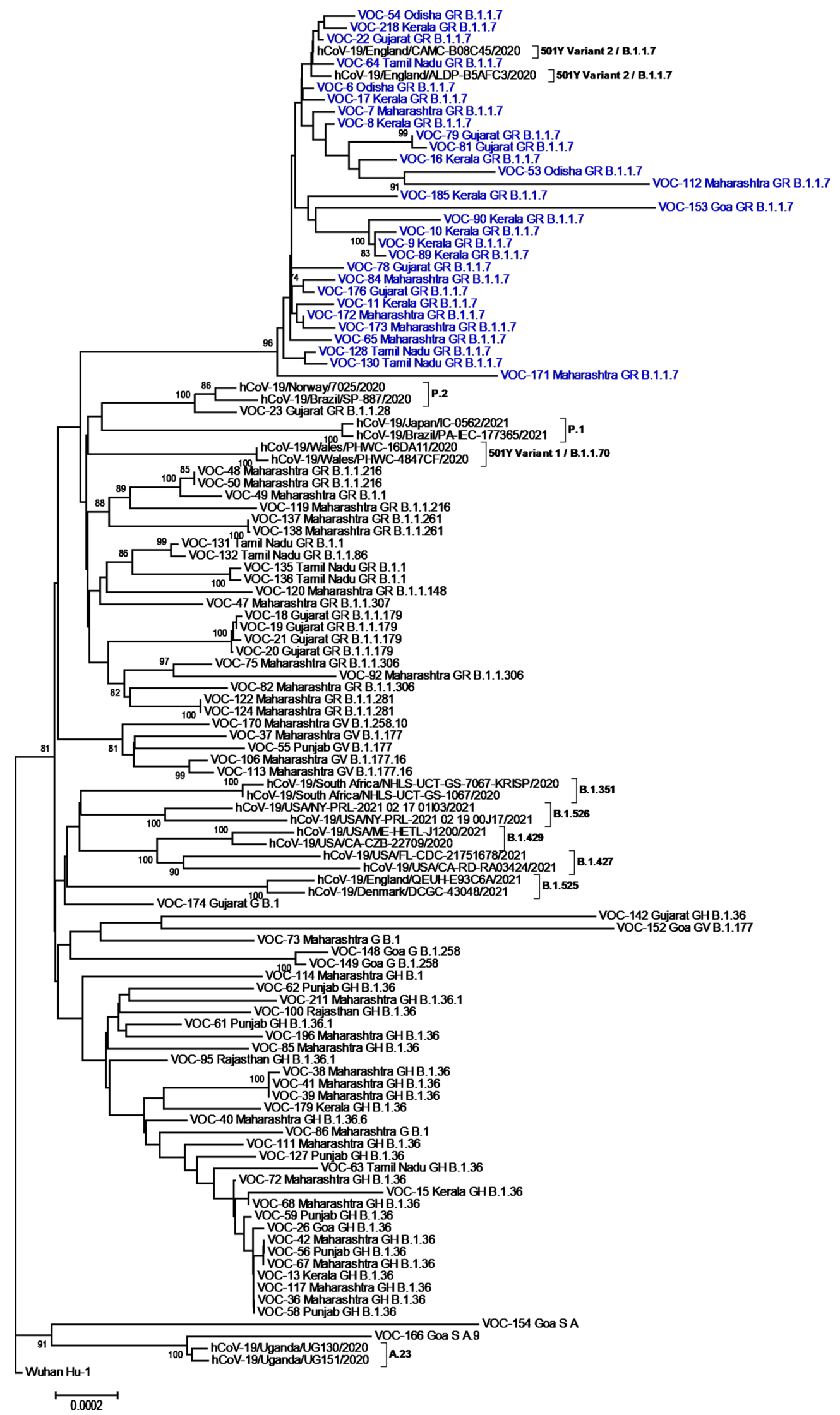


4Fig. 1 Phylogenetic tree of selected whole-genome sequences of SARS-CoV-2 in this study $(n=93)$ along with Wuhan Hu- 1 and other reference strains of major variants using the Neighbor-Joining method. Strains identified as the UK variants are shown in blue color

returnees across different states in India for carrying out the SARS CoV-2 genomic analysis, with specific emphasis on identification of the UK variant. At ICMR-NIV, Pune 385 samples received from December 23 January 14, 2021, were tested by TaqPath ${ }^{\mathrm{TM}}$ COVID-19 Combo Kit-S gene and Next-generation sequencing (NGS) using the Ion Torrent platform. The $\mathrm{S}$-gene deletion at genomic positions 21,765-21,770, corresponding to residues 69-70 in the spike protein of the UK variant and other variants carrying this deletion/ mutation, may cause some RT-PCR assays targeting the S-gene to produce a negative result (S-gene dropout) [1]. The tri target (ORF1ab, N, S) COVID-19 TaqPath assay from Thermo Fischer has been reported by the UK investigators to have S-gene dropout for this deletion [1]. The S-gene is generally not used by itself for detection of the virus hence S-gene drop-out is unlikely to cause an overall false-negative result for SARS-CoV-2 detection. However, S-gene drop-out can be used as a proxy measure of the incidence of the variant. Further, all the COVID-19 positive and S-gene drop-out samples were analysed for the whole genome using Ion Torrent NGS platform. The phylogenetic tree was constructed using MEGA v.6 [3], employing the Neighbor-Joining method with "Maximum Composite Likelihood" as the substitution model and 1,000 bootstrap replications. For each whole-genome sequence, the Global Initiative on Sharing All Influenza Data (GISAID) Clade Assignment, was done using CoVsurver: Mutation Analysis of hCoV-19 (https://www.gisaid.org/epiflu-applications/ covsurver-mutations-app/). For lineage assignment, the web application, Phylogenetic Assignment of Named Global Outbreak LINeages (PangoLIN) COVID-19 Lineage Assigner (https://pangolin.cog-uk.io/), was implemented [4].

There are at least 3 designations for the nomenclatures of the SARS-CoV-2 strains in use [5]. Studies that describe the geographic distribution of SARS-CoV-2 clades and variants across the world using whole-genome sequencing have revealed that the GISAID clades G, GH and GR were predominant particularly in Europe and North America. In the United Kingdom, two new SARS-CoV-2 lineages with the N501Y mutation in the receptor-binding domain (RBD) of the spike protein spread rapidly [6]. An earlier 501Y lineage without amino acid deletion $\Delta 69 / \Delta 70$, circulated mainly between early September and mid-November, and was $\sim 10 \%$ more transmissible than the $501 \mathrm{~N}$ lineage. Further, a 501Y lineage with amino acid deletion $\Delta 69 / \Delta 70$, circulated since late September, and was $\sim 75 \%$ more transmissible than the $501 \mathrm{~N}$ lineage. These newly emerged SARS-CoV-2 variants within the GISAID GR clade were referred to as UK-variant
1 and variant 2 (VOC 202012/01) (B.1.1.7 in the PangoLIN lineage classification). In addition to VOC 202012/01, South Africa reported another SARS-CoV-2 variant within the GISAID GH clade, designated as 501.V2 (B.1.351 in PangoLIN lineage classification), during October 2020, which is also of potential concern [1]. Additionally, P.1 and P.2 lineages, aliases of lineage B.1.1.28.1 and B.1.1.28.2 respectively, within the GISAID GR clade, were first identified in Brazil during December 2020 [1]. The South Africa and Brazilian P.1 variants possessed E484K and N501Y along with K417N/T in the spike protein RBD which are of biological significance. The Brazilian P.2 variant had only E484K.

Asian countries, including India had the early diverged clades namely $\mathrm{S}, \mathrm{L}$ and $\mathrm{V}$ in addition to the G-type of clades [7]. The epidemiology of SARS-CoV-2 in India over the period revealed that the predominant clades (PangoLIN/ GISAID) circulating in India are the B.1.1.32/GR, B.6/O, B.1/G, B.1.1/GR, B.1.113/GH and B.1.1.8/GR [8]. To monitor the likely introductions of the newer SARS-CoV-2 variants, ICMR-NIV, Pune received 385 samples for the genomic analysis from fifteen states of India, till January 14,2021 , There were 170 (44.2\%) females and 215(55.8\%) males, the median age of the cases was 37 years [Inter-quartile range (IQR); 28-50 years]. Of all 385 subjects, 33(8.6\%) were symptomatic, mostly related to upper respiratory illness. All of the cases were quarantined in a separate health facility by their respective state health authorities. Of the total referred cases, 21 of 385 (5.5\%) were from the UK returnee close contacts. The median days of collection of respiratory specimen from the day of arrival from UK were 8 days (IQR 0-13 days). TaqPath COVID-19 RT-PCR result showed that 88 of 385 (22.9\%) were COVID-19 negative and 212(55.1\%) were COVID-19 positive. Among the 212 positives, 78 (36.8\%) positives showed S-gene drop out in the TaqPath COVID-19 RT-PCR assay.

Because of either insufficient viral RNA for genomic analysis or insufficient sample, the sequencing could not be performed for 58 COVID-19 of the 212 positive samples. Additionally, fourteen samples were rejected due to nonavailability of travel/clinical history. As of January 14, 2021, the whole genome sequencing and analysis were undertaken for totally 140 samples of COVID-19 positive and S-gene drop-outs. Sequences from 29 samples could not be considered due to low quality and 18 were excluded from the phylogenetic analysis due to short sequence length. Out of the 93 sequences considered (Table S1), 30 (29 UK returnees and one contact) were positive for the SARS CoV-2 UK variant and belonged to lineage B.1.1.7 within the clade GR (Fig. 1). Five sequences, three from Maharashtra and one from Punjab and Kerala each were classified as B.1.177 (GV clade) which to the best of our knowledge are among the first reported GV strains in India. Among the detected cases 
of SARS CoV-2 UK variant, 10 were from Kerala, 8 were from Maharashtra, 5 from Gujarat, 3 each from Odisha and Tamil Nadu and one from Goa (Fig. 2). While comparing the result of NGS (gold standard) with TaqPath RT-PCR (S-gene drop-out), the sensitivity of TaqPath for detecting the SARS CoV-2 UK variant was found to be $94.12 \%$ [Confidence Interval (CI): 80.9-98.3\%] and specificity 98.28\% [Confidence Interval (CI): 90.8-99.7\%] with an overall diagnostic accuracy of $96.7 \%$ [Confidence Interval (CI): 90.8-98.8\%].

Most of the new candidate vaccines are based on the spike protein sequence. Therefore, it is essential to monitor variations in the spike protein among the circulating SARS-CoV-2 strains and assess possible antigenic changes. However, available evidences and report from other different mutations in the spike RBD do not appear to have a significant impact on the ability of antibodies in the sera from subjects vaccinated with mRNA-based vaccine to neutralise such variants [https://www.ema. europa.eu/en/documents/assessment-report/comirnatyepar-public-assessmentreport_en.pdf]. Studies towards understanding the infectivity and pathogenicity of the UK SARS CoV-2 variant in India would however need to be pursued. There is currently a lack of evidence indicating that the UK SARS CoV-2 variant virus mentioned in this report is widespread in India. Given that sequencing performed still is in low numbers, therefore it is probable that there may be substantial under-detection. Nonetheless considering the extent to which the new variants of concern are circulating, efficient implementation of non-pharmaceutical measures in response to the epidemiological situation remains essential. Hygiene rules for prevention of transmission of SARS-CoV-2 remain effective even for the UK-variant and other variants of concern. This would

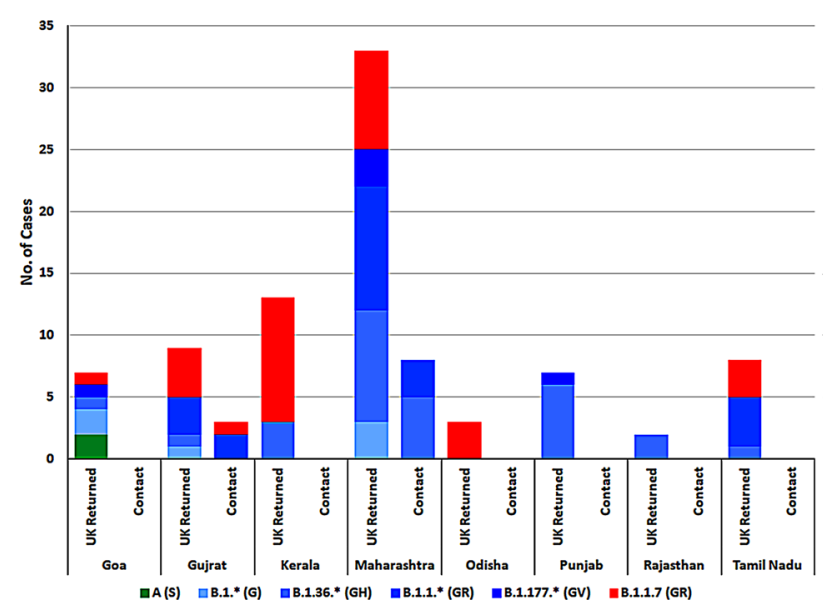

Fig. 2 Distribution of the variants in the different Indian states based on the sequences of 93 samples from the UK returnees and few contacts be vital until and unless vaccination is shown to mitigate their impact.

Supplementary Information The online version contains supplementary material available at https://doi.org/10.1007/ s15010-021-01617-6.

Acknowledgements Authors gratefully acknowledge National Influenza center staff: Dr M Das, Mr Y Balakartik, Ms K Korabu, Mrs K Iyengar, Mr Shelkande, Dr S. Salve, Dr V. Malik, Ms P. Malsane, Ms P. Shinde, Ms S. Bhorekar, Mr H. Kengle, A. Awhale Mr V. Awatade, Mr V kishty, Mr K Suryavanshi, Mr S Zakiuddin, Mr A Titkare and Mr. A.M.Walimbe, Bioinformatics Group, ICMR-NIV, Pune. The authors acknowledge the encouragement and support extended by Prof. (Dr) Balram Bhargava, Secretary to the Government of India, Department of Health Research, Ministry of Health and Family Welfare, and Director-General, Indian Council of Medical Research (ICMR), New Delhi, and Dr. Samiran Panda and Dr. Nivedita Gupta, Division of Epidemiology \& Communicable Diseases, ICMR, New Delhi.

Author contributions Conceived-VAP, SC and PA; Experiments: VV, SYJ, US; Analysis: VAP, SC, SMJ, SB, MLC; Writing: VAP, $\mathrm{SC}, \mathrm{SB}$.

Funding Authors acknowledge the Department of Health Research, Ministry of Health \& Family Welfare, Government of India, New Delhi, for financial support.

Availability of data and material (data transparency) Available.

\section{Declarations}

Conflict of interest None.

Ethics approval Waiver.

Consent for publication All authors agree for publication.

\section{References}

1. ECDC. Risk Assessment: Risk related to spread of new SARSCoV-2 variants of concern in the EU/EEA - first update. Available at https://www.ecdc.europa.eu/en/publications-data/ covid-19-risk-assessment-spread-new-variants-concern-eueeafirst-update. Accessed 2 Mar 2021.

2. Kupferschmidt K, Fast-spreading UK. virus variant raises alarms. Science. 2021;371(6524):9-10.

3. Tamura K, Stecher G, Peterson D, et al. MEGA6: Molecular Evolutionary Genetics Analysis version 6.0. Mol Biol and Evol. 2013;30 2725-29.

4. Rambaut A, Holmes EC, Hill V, et al. A dynamic nomenclature proposal for SARS-CoV-2 to assist genomic epidemiology. Nat Microbiol. 2020;5:1403-7.

5. Alm E, Broberg EK, Connor T, et al. The WHO European Region sequencing laboratories and GISAID EpiCoV group. Geographical and temporal distribution of SARS-CoV-2 clades in the WHO European Region, January to June 2020. Euro Surveill. 2020; 25(32):pii=2001410. 
6. Leung K, Shum MHH, Leung GM, et al. Early transmissibility assessment of the N501Y mutant strains of SARS-CoV-2 in the United Kingdom, October to November 2020. Euro Surveill. 2021; 26(1):pii=2002106.

7. Potdar V, Cherian SS, Deshpande GR, et al. Genomic analysis of SARS-CoV-2 strains among Indians returning from
Italy, Iran \& China, Italian tourists in India. Indian J Med Res. 2020;151:255-60.

8. Potdar V, Vipat V, Ramdasi A, et al. Phylogenetic classification of the whole genome sequences of SARS-CoV-2 from India and evolutionary trends. Indian J Med Res. 2021; In press. 